\title{
Association study and mutation sequencing of genes on chromosome 15q11-q13 identified GABRG3 as a susceptibility gene for autism in Chinese Han population
}

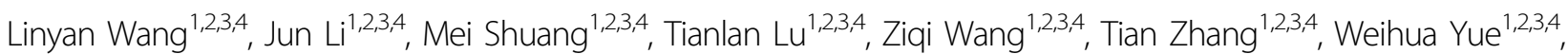 \\ Meixiang Jia ${ }^{1,2,3,4}$, Yanyan Ruan ${ }^{1,2,3,4}$, Jing Liu' ${ }^{1,2,3,4}$, Zhiliu Wu $u^{1,2,3,4,5}$, Dai Zhang ${ }^{1,2,3,4,6,7}$ and Lifang Wang ${ }^{1,2,3,4}$
}

\begin{abstract}
Cytogenetic studies suggested that chromosome 15q11-q13 might be a candidate region that increases the risk of autism. Previous association studies in Caucasian populations identified the risk variants of genes in this region. However, the association of these genes with autism in Chinese Han population remains unclear. Herein, 512 autism trios were utilized for a family-based association study of 41 tag single nucleotide polymorphisms (SNPs) in this region to explore the association between protein-coding genes on chromosome 15q11-q13 and autism in Chinese Han population. Furthermore, we sequenced these autism-related genes to detect rare variants in 512 autism trios and 575 healthy controls. Our results showed that the $C$ allele of rs7180500 in GABRG3 was a risk variant for autism ( $p=$ 0.00057). The expression quantitative trait loci (eQTL) analysis revealed that the $C$ allele of rs 7180500 might be associated with the expression of GABRG3 in the cerebellum (Braineac: $p=0.0048$; GTEx: $p=0.0010$ ). Moreover, the sequencing identified two rare variants rs201602655 (p.Val233Met) and rs201427468 (p.Pro365Ser) in GABRG3 and six rare variants in GABRB3 in autistic patients. Among these variants, rs201602655 (p.Val233Met) in GABRG3 were observed in 9 of 512 autistic children and 2 of 575 healthy controls (Pearson $X^{2}$-test, $X^{2}=5.375, p=0.020$ ). The functional prediction indicated that rs201602655 (p.Val233Met) might be deleterious. Thus, these findings demonstrated that GABRG3 might contribute to the pathogenesis of autism in Chinese Han population.
\end{abstract}

\section{Introduction}

Autism is a severe neurodevelopmental disorder with a typical onset before 3 years of age. The condition is primarily characterized by three abnormal symptoms: impairment in social interaction, the deficit in

Correspondence: Jing Liu (ljyuch@bjmu.edu.cn) or Zhiliu Wu

(zhiliu_wu@bjmu.edu.cn) or Lifang Wang (lifangwang@bjmu.edu.cn)

${ }^{1}$ Peking University Sixth Hospital, Beijing 100191, China

2Peking University Institute of Mental Health, Beijing 100191, China

Full list of author information is available at the end of the article.

These authors contributed equally: Linyan Wang, Jun Li, Mei Shuang communication, and repetitive and restricted behaviors or interests. The prevalence of this disorder in the worldwide population is estimated at $\sim 1 \%$. Genetic factors were considered to play a critical role in the etiology of autism. Family and twin studies indicated that autism was highly hereditary ${ }^{1,2}$. Reportedly, the concordance in autism of monozygotic twins was $70 \%-90 \%$ as compared to $~ 10 \%$ in dizygotic twins ${ }^{3}$. However, the role of genetic factors in the pathogenesis of autism remains unclear.

Chromosome 15q11-q13 has been identified as a candidate region that increases the risk of autism ${ }^{4}$. This

\section{(c) The Author(s) 2018}

(c) (i) Open Access This article is licensed under a Creative Commons Attribution 4.0 International License, which permits use, sharing, adaptation, distribution and reproduction in any medium or format, as long as you give appropriate credit to the original author(s) and the source, provide a link to the Creative Commons license, and indicate if changes were made. The images or other third party material in this article are included in the article's Creative Commons license, unless indicated otherwise in a credit line to the material. If material is not included in the article's Creative Commons license and your intended use is not permitted by statutory regulation or exceeds the permitted use, you will need to obtain permission directly from the copyright holder. To view a copy of this license, visit http://creativecommons.org/licenses/by/4.0/. 
region contains several critical genes, such as $\mathrm{GABA}_{\mathrm{A}}$ receptor genes cluster, $U B E 3 A$ and $C Y F I P 1$, which might be correlated with the development and function of the brain $^{5-7}$. The postmortem of autistic individuals revealed a reduced expression of GABRB3, GABRA5, and/or GABRG3, which was detected in several specific brain regions, such as the superior frontal cortex, parietal cortex, and cerebellum ${ }^{8}$. Positron emission tomography (PET) study further confirmed the reduced level of $\mathrm{GABA}_{\mathrm{A}}$ receptor $\alpha 5$ subunit in the brains of autistic patients ${ }^{9}$. In addition, deficiency of $\mathrm{GABA}_{\mathrm{A}}$ receptor genes cluster might be involved in autism-like behaviors. Gabrb3 $^{-1-}$ mice exhibited significant impairments in activities, including sociability, social novelty, and nesting, as well as tactile and heat hypersensitivity. These features were similar to the symptoms of partial autistic children $^{10,11}$. Gabra5 ${ }^{-1-}$ mice exhibited reduced social contact as well as the alterations in electroencephalograph (EEG) patterns, which were reported in autistic individuals $^{12,13}$. Moreover, mice with overexpression of $U b e 3 a$, Snprn, or Cyfip1 showed autism-like social deficits and repetitive self-grooming behavior ${ }^{14-18}$. Thus, these findings indicated that the dysfunction of genes on chromosome 15q11-q13 might play a crucial role in the pathogenesis of autism.

Previous association studies demonstrated that genes on chromosome $15 \mathrm{q} 11-\mathrm{q} 13$, especially $\mathrm{GABA}_{\mathrm{A}}$ receptor genes cluster, might be autism susceptibility genes. Transmission disequilibrium tests (TDT) for 16 single nucleotide polymorphisms (SNPs) in $\mathrm{GABA}_{\mathrm{A}}$ receptor genes cluster indicated that 2 SNPs in GABRG3 were nominally associated with autism in Caucasians ${ }^{19}$. Other family-based studies detected nominally associated SNPs in GABRB3 and GABRA5 with autism in Europeans and Koreans $^{20-22}$. Furthermore, a recent case-control study indicated that several SNPs and haplotypes in GABRB3 were significantly associated with Asperger syndrome ${ }^{23}$, a subgroup of autism spectrum disorder (ASD). For other genes, such as SNRPN, CYFIP1, and ATP10A, a few SNPs or haplotypes were found to be nominally associated with autism in Europeans ${ }^{20,24-26}$.

In addition to common variants, rare variants might contribute to high heritability of autism. Rare and especially de novo genetic variations are known to affect liability $^{27-30}$. Some patients with various neurodevelopmental disorders carried 15q11.2 duplication and deletion of CYFIP1, NIPA2, and NIPA1 $1^{31,32}$. Moreover, several rare inherited variants in GABRB3 were detected in patients affected with $\mathrm{ASD}^{33}$. Moreover, another study reported that a rare novel maternal transmission variant of GABRB3 was associated with autism ${ }^{34}$.

In this study, we hypothesized that single nucleotide polymorphisms (SNPs) and rare mutations in the genes related to ASD and neurodevelopment in chromosome 15q11-q13 region were associated with autism. To explore the association of these genes on chromosome 15q11-q13 with autism, we performed a family-based association study between 10 protein-coding genes and autism in 512 nuclear trios of Chinese Han descent. Furthermore, we sequenced the genes on chromosome 15q11-q13 region to detect the rare variants that might contribute to the pathogenesis of autism.

\section{Materials and methods \\ Ethics statement}

This study was approved by the Ethics Committee of Peking University Sixth Hospital (China). All participants provided written informed consent to participate in this study. The informed consents of children were obtained from their legal guardians. All protocols were performed in accordance with the approved guidelines.

\section{Subjects}

All subjects were of Chinese Han origin and recruited at Peking University Sixth Hospital China. The patients fulfilled the criteria of the Diagnostic and Statistical Manual of Mental Disorders, Fourth Edition (DSM-IV) for autistic disorder. Children with Asperger syndrome, Rett syndrome, pervasive developmental disorder not otherwise specified (PDD-NOS), fragile $\mathrm{X}$ syndrome, tuberous sclerosis, a previously identified chromosomal abnormality, dysmorphic features, or any other neurological condition were excluded from the study. Individuals with other familial inherited diseases or severe psychiatric disorders were also excluded. The diagnosis of autism was established by two senior psychiatrists. Autism Behavior Checklist $(\mathrm{ABC})$ and Childhood Autism Rating Scale (CARS) were used to evaluate the clinical features of children $^{35,36}$. Children with $\mathrm{ABC}$ score $\geq 53$ and CARS scores $\geq 35$ were included. A total of 512 autism nuclear trios were included in this study, of which, 449 were males and 63 were females (ratio of male: female, 7:1). The median age of the children at the time of diagnosis was 4.5 (range, 3-14) years.

We recruited age- and sex-matched healthy controls from Peking University Sixth Hospital China. A total of 575 individuals, including 480 males and 95 females, were recruited. The ratio of male to female was about 5:1. The age of healthy controls ranged from 3 to 12 years.

\section{SNPs selection and genotyping}

We selected 10 protein-coding genes on 15q11-q13, which were related to ASD and brain development. The genotype data of all SNPs for Chinese Han general population in Beijing $(\mathrm{CHB})$ was downloaded from the Genome Variation Server 147 (http://gvs.gs.washington. 


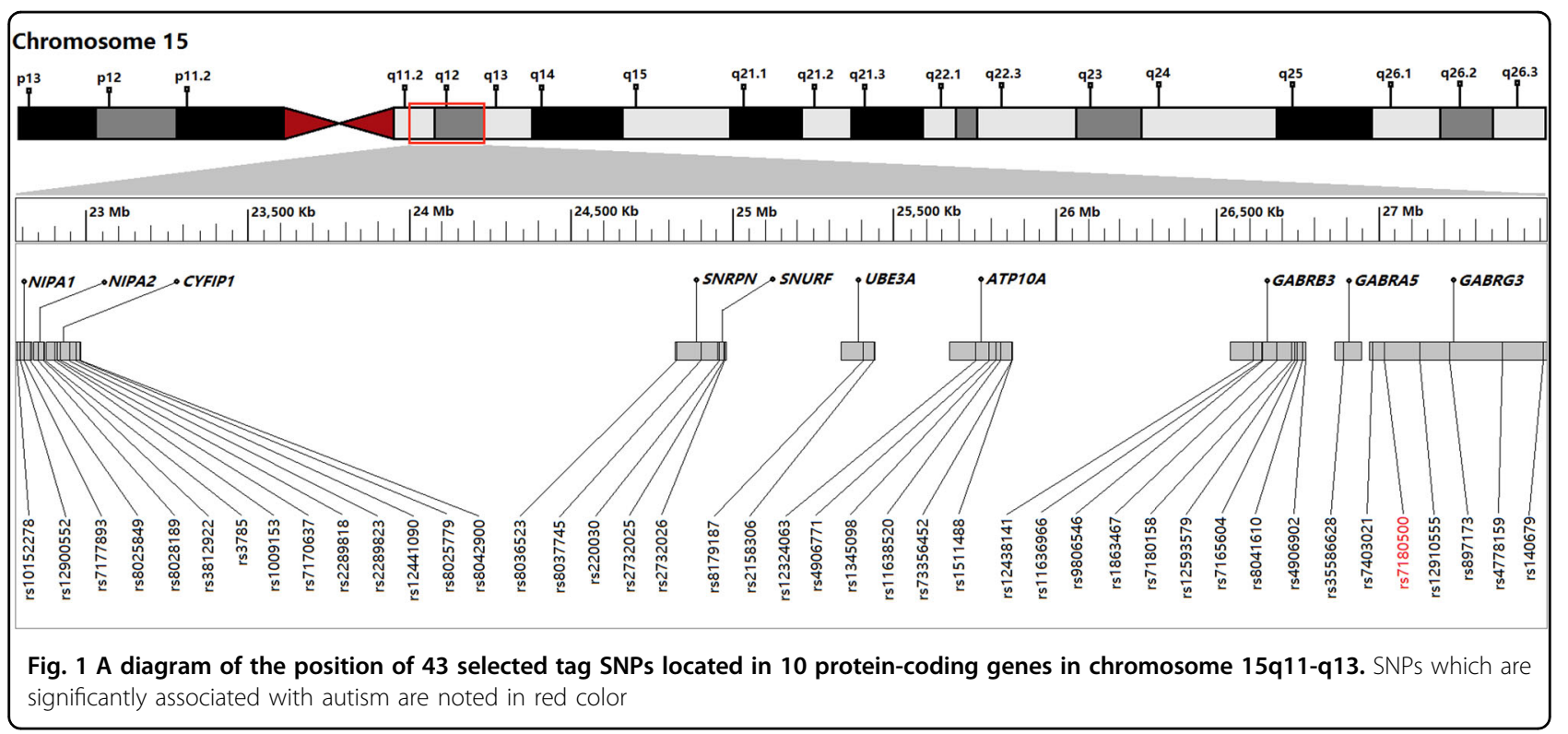

edu/GVS147) and the dbSNP in NCBI (http://www.ncbi. nlm.nih.gov/SNP/). The principles of tag SNPs selection were as follows: the minor allele frequency (MAF) value of the selected SNP should be greater than 0.05; positiveassociated SNPs reported in other ethnic population and genome-wide association study (GWAS) data with autism, autism spectrum disorders type were selected; SNPs located in the functional regions of genes, such as the promoter, $5^{\prime}$ untranslated region (UTR), exons and $3^{\prime}$ UTR were preferential. After utilizing the Tagger module in Haploview version 4.2, a total of 43 tag SNPs of 10 genes were included in this study (Fig. 1). The $p$-values of Hardy-Weinberg Equilibrium (HWE) of all tag SNPs in the CHB general population were $>0.05$.

Genomic DNA was extracted from the blood using a Qiagen QIAamp DNA Mini Kit (Qiagen, Hilden, Germany). The genotyping of SNPs in 10 protein-coding genes was performed using the Agena Bioscience platform (Agena Bioscience, San Diego, USA), which is based on the matrix-assisted laser desorption/ionization time-offlight (MALDI-TOF) primer extension assay ${ }^{37}$. Singlebase primer extension with mass-modified terminators increased the plexing efficiency and flexibility of the MassARRAY system ${ }^{38}$. The DNA products for each SNP were prepared according to the three primary steps: DNA amplification, shrimp alkaline phosphatase (SAP) reaction and extension. All primers were designed according to the sequence of the forward strand provided by the hg19 assembly. These primers are listed in Supplementary Table S1. Subsequently, the products were dispensed into the SpectroCHIP array and the spectra of products for each SNP were acquired using the platform's mass spectrometer. Next, we randomly selected $10 \%$ samples to confirm the genotyping results using Sanger sequencing.

\section{Target genes capture and sequencing}

A customized capture array (NimbleGen, Roche) was designed to enrich the genes on chromosome 15q11-q13 (including NIPA1, NIPA2, CYFIP1, SNRPN, SNURF, UBE3A, ATP10A, GABRB3, GABRA5, and GABRG3) according to the Build GRCh 37 assembly genome annotation of NCBI. The sequencing regions included all exons, $1 \mathrm{~Kb}$ upstream of the transcription start site, and $3^{\prime} \mathrm{UTR}$. To avoid the nonspecific binding of genomic elements to capture arrays, RepeatMasker (http://ftp.genome. washington.edu/RM/RepeatMasker.html) was used to exclude highly repetitive elements from the probe. The method similar to the WindowMasker program was used to identify these regions. Genomic DNA was captured on a NimbleGen' array following the manufacturer's protocols. Briefly, the genomic DNA of participants was fragmented to a size of $200 \mathrm{bp}$ by ultrasonoscope. The DNA was sheared by sonication and adaptors were ligated to the resulting fragments. Subsequently, the extracted DNA was amplified by ligation-mediated polymerase chain reaction (PCR), purified, and hybridized to the capture array at $42^{\circ} \mathrm{C}$ using the manufacturer's buffer. The resulting fragments were purified and subjected to DNA sequencing on the Illumina HiSeq2500 Analyzers platform according to the manufacturer's protocol (Fig. S1).

\section{Data filtering and analyses}

Illumina Pipeline (version 1.3.4) was used to generate primary data containing image analyses, error estimation 
and base calling. Indexed primers were used to identify different reads in the raw data. These reads were accepted when they matched to the theoretical adapter indexed sequences, as well as the theoretical primer indexed sequences with a maximum of three mismatches. Then, we excluded the unqualified sequences including lowquality reads, defined as those containing $>5 \% \mathrm{Ns}$ in the read length, reads with $>30 \%$ bases with a quality score $<$ Q20, and adapter-contaminated read pairs including the indexed sequence. The remaining sequences were termed as clean reads for analysis.

Burrows-Wheeler Aligner (BWA) ${ }^{39}$ was employed to align the clean reads to the human reference genome from the NCBI database (build GRCh37). Picard tool was used to convert the sequence alignment files. Variant calling was performed by the Genome Analysis Toolkit $(\text { GATK })^{40}$. The previously identified SNPs were determined using the NCBI dbSNP. Based on the Human Gene Mutation Database at the Institute of Medical Genetics in Cardiff (HGMD, http://www.ghmd.cf.ac.uk/) or previous literature, we identified the known disease-causing mutations. The data analysis is schematically represented in Fig. S2. In addition, SIFT (http://sift.jcvi.org), PolyPhen-2 (http://genetics.bwh.harvard.edu/pph2/), and Mutation Taster (http://www.mutationtaster.org/) were used to predict the pathogenicity of the detected mutations.

\section{Sanger sequencing}

The mutations detected by targeted sequencing were further confirmed using Sanger sequencing. The primer design tool (https://www.ncbi.nlm.nih.gov/tools/primerblast/) was used to design the specific primers (Table S2). PCR was performed using the $2 \times$ EasyTaq PCR SuperMix (including EasyTaq DNA polymerase, dNTP and buffer) (TransGen Biotech, Beijing, China). The optimal annealing temperature for PCR was $62^{\circ} \mathrm{C}$. The DNA sequencing was performed using a BigDye Terminator Cycle Sequencing Ready Reaction Kit with AmpliTaq DNA polymerase. The PCR fragments were separated by electrophoresis on an ABI PRISM 377-96 DNA Sequencer (Applied Biosystem, Foster City, USA).

\section{Statistical analysis}

The power of detection of risk alleles was estimated by Quanto software version 1.2.4 (http://biostats.usc.edu/ software). For the identified significant risk alleles, the relative risk was set to values calculated from the previously described formula for family-based samples ${ }^{41} \cdot \chi^{2}$ test was used to analyze the deviations from the HWE for genotype frequency distributions. Single marker association tests were performed using the family-based association test (FBAT) program version 2.0.3 (http://www. biostat.harvard.edu/fbat/default.html). This program implemented a generalized statistical score to perform various transmission disequilibrium tests (TDTs). Additive and recessive inheritance models were examined utilizing these tests. The genotypes of families with Mendelian errors were detected and reset to zero by FBAT. Furthermore, Bonferroni correction was performed to reduce the rate of type I errors. The significance level was set at $p<\alpha / n(\alpha=0.05)$. All $p$-values calculated by the FBAT were two-sided. Haploview version 4.2 (http://www.broad.mit.edu/mpg/haploview/) was used to calculate the ratio of transmission to untransmission ( $\mathrm{T}$ : U) for alleles of each selected SNP.

The frequencies of rare variants were compared between the patients and controls using Pearson $\chi^{2}$-test when the calculated minimum expected count was $>5$. While Continuity Correction test were performed when the calculated minimum expected count was $>1$ and $<5$. The significance was set at $p<0.05$ (two-sided).

\section{Expression quantitative trait loci (eQTL) analysis of significantly associated SNPs and expression pattern in human brain}

Two online databases were used to analyze the eQTL effects of the risk alleles of the associated SNPs. The Brain eQTL Almanac (Braineac) (http://www.braineac.org/) ${ }^{42}$ and Genotype-Tissue Expression (GTEx) database (http:// www.gtexportal.org $/)^{43}$ provided the eQTL data for ten primary brain regions with significantly associated SNPs, respectively. These online databases aided in exploring whether one or more risk SNPs were operating as eQTL in brain regions. Moreover, the GTEx database provided the data for genes in 53 human tissues or cells. The Human Brain Transcriptome (HBT) databases (http:// hbatlas.org/pages/hbtd) provided a dynamic expression of significantly associated genes during the development and adulthood in different regions of the brain.

\section{In silico analysis of SNPs and mutations}

We explored the annotations of SNPs and detected the mutations using HaploReg v4.1 (http://compbio.mit.edu/ HaploReg) which can identify the state of chromatin, conservation, and regulatory motif alterations of risk variants and $\mathrm{SNPs}^{44}$. MiRWalk 2.0 (http://zmf.umm.uniheidelberg.de/apps/zmf/mirwalk2/) and TargetScan (http://www.targetscan.org/vert_71/) were used to predict whether the detected SNPs or variants were the target regions of miRNAs. rVarBase (http://rv.psych.ac.cn/index. do) ${ }^{45}$ and JASPAR (http://jaspar.genereg.net/) were used to explore whether the associated SNPs and mutations were regulatory SNPs and transcription factors (TFs). Furthermore, Promoter 2.0 Prediction Server (http:// www.cbs.dtu.dk/services/Promoter/) and Eukaryotic 
Promoter database (http://epd.vital-it.ch/) were used to identify whether the variants were located in the promoter regions.

\section{Results \\ Quality control}

Genotypes of all SNPs were clustered clearly using the Agena Bioscience platform. The call rate of each genotype was ensured to be $>0.95$. Among the 43 selected tag SNPs, 2 SNPs (rs7170637 and rs11636966), which are common risk variants in Europeans with ASD, were identified as rare variants in our samples $(\mathrm{MAF}<0.05)$. Thus, $41 \mathrm{tag}$ SNPs were qualified for analysis. The allele frequencies of these 41 tag SNPs were displayed in Table 1. Among the 512 autism trios, the power to detect the potential risk alleles for the 41 selected SNPs ranged from 0.6 to 0.99 . None of the genotype distributions of these tag SNPs in unaffected parents deviated from HWE (Table S2). The genotyping concordance rate for Agena Bioscience platform and direct Sanger sequencing was $>99 \%$.

\section{SNP association analyses}

Under the additive model, the single SNP association tests demonstrated that the $\mathrm{C}$ allele of rs7180500 in GABRG3 was preferentially transmitted from unaffected parents to affected offspring (rs7180500: $\mathrm{C}>\mathrm{A}, Z=3.488$, $p=0.00057$ ) (Table 1). The significance of this result persisted even after the Bonferroni correction $(p=\alpha / n=$ $0.05 / 41=0.0012$ ). Under the recessive model, the $C$ allele of rs7180500 in GABRG3 also displayed a nominal association with autism (rs7180500: $\mathrm{C}>\mathrm{A}, Z=2.798, p=$ 0.0052). The $\mathrm{G}$ allele of rs 4906902 in GABRB3 was a risk variant, and the $\mathrm{C}$ allele of rs4906771 in ATP10A was a protective variant after Bonferroni correction (rs4906902: A $>$ G, $Z=3.441, \quad p=0.00065 ; \quad r s 4906771: \quad \mathrm{T}>\mathrm{C}, \quad Z=$ $-3.395, p=0.00069$ ) (Table S4). Moreover, 7 SNPs (rs3812922 in NIPA2, rs8037745 in SNRPN, rs4906771 and rs1345098 in ATP10A, rs12438141, rs1863467, and rs4906902 in GABRB3) displayed nominal association with autism under the additive model in our samples $(p<$ 0.05) (Table 1). Nine SNPs (rs8025849 in NIPA1, rs3812922 in NIPA2, rs1009153 and rs2289818 in CYFIP1, rs8037745 in SNRPN, rs12438141 and rs1863467 in GABRB3, rs7403021 and rs7180500 in GABRG3) were nominally associated with autism under the recessive model $(p<0.05)$ (Table S4).

\section{Discovery and validation of rare variants}

In the discovery phase, we performed targeted sequencing for the genes (including exonic coding regions and transcriptional regulation regions) on chromosome 15q11-q13 in 96 patients affected with autism. The probe coverage reached $98.1 \%$. The average ratio of the target capture was $>80 \%(200 \times$ depth). We detected two rare variants rs201602655 (p.Val233Met) and rs201427468 (p. Pro365Ser) in GABRG3 in 2 autistic children and six rare single nucleotide variants (c. $-693 \mathrm{~A}>\mathrm{T}$, c. ${ }^{*} 417 \mathrm{C}>\mathrm{T}$, c. $* 704 \mathrm{~A}>\mathrm{T}, \quad$ c.*1730G $>\mathrm{A}, \quad$ c. ${ }^{*} 2583 \mathrm{C}>\mathrm{T}, \quad$ c.*3536T $\left.>\mathrm{C}\right)$ in GABRB3 in 6 of 96 patients affected with autism. These variants (all heterozygote) were validated by Sanger sequencing (Fig. 2 and Fig. S3).

Then, we expanded the sample size to 512 patients with autism and their parents in the validation phase. As for rs201602655 (p.Val233Met) in GABRG3, one autistic child $(1 / 512)$ presented this de novo heterozygous mutation and 8 children affected with autism (8/512) were inherited from their parents (four were maternal and the others were paternal, all heterozygotes). Furthermore, we sequenced this mutation (rs201602655, p.Val233Met) in 575 age-matched healthy controls and found that 2 controls carried this heterozygous variant. The frequency of rs201602655 (p.Val233Met) in GABRG3 in autistic children was significantly higher than that in healthy controls (9/512 vs. $2 / 575$, Pearson $\chi^{2}$-test, $\left.\chi^{2}=5.375, p=0.020\right)$ (Table 2).

Another rare variant rs201427468 (p.Pro365Ser) in GABRG3 was detected in 3 autistic children (3/512) (one was de novo, and the other two were inherited from fathers, all heterozygotes). One healthy control also carried this mutation (1/575, heterozygote). The frequency of heterozygote of rs201427468 (p.Pro365Ser) between patients and controls demonstrated no significant difference ( $3 / 512$ vs. $1 / 575$, continuity correction $\chi^{2}=0.382, p$ $=0.537)$ (Table 2).

All of the six rare single nucleotide variants (c. $-693 \mathrm{~A}>\mathrm{T}, \quad$ c." $417 \mathrm{C}>\mathrm{T}, \quad$ c."704A $>\mathrm{T}, \quad$ c." $1730 \mathrm{G}>\mathrm{A}, \quad$ c. "2583C $>\mathrm{T}$, c."3536T $>\mathrm{C}$ ) in GABRB3 detected in the discovery phase were inherited. When the sample size was expanded to 512 autistic children, each variant was detected only in one autistic child, respectively (Fig. S3 and Table S5).

\section{eQTL effects of the significantly associated SNPs and expression profile of GABRG3 in human brain}

Two online eQTL databases revealed that the $\mathrm{C}$ allele of rs7180500 might be associated with the expression level of GABRG3 in the cerebellum (Braineac: $p=0.0048$; GTEx: $p=0.0010$ ) (Fig. 3). The data from Braineac showed that rs4906771 in ATP10A might exert potential eQTL effects on the frontal cortex. However, a similar result was not obtained using the GTEx databases (Braineac: $p=0.022$; GTEx: $p=0.12$ ) (Fig. S4 and Table S5).

Furthermore, the RNA-seq data from GTEx revealed that the expression of GABRG3 was higher in several brain regions, such as the cerebellum and hypothalamus, as compared to other human tissues. The HBT database showed an increased GABRG3 expression in the hippocampus and other brain regions during developmental 
Table 1 Results of association analyses between 41 tag SNPs in chromosome 15q11-q13 in 512 trios by FBAT under an additive model

\begin{tabular}{|c|c|c|c|c|c|c|c|c|c|c|}
\hline Gene symbol & Marker & Chromosome & Allele & Afreq & Fam & $T: U^{a}$ & S-E (S) & $\operatorname{Var}(\mathrm{S})$ & $Z$ & $P^{\mathrm{b}}$ \\
\hline \multirow[t]{8}{*}{ NIPA1 } & rs10152278 & 15:22786139 & G & 0.571 & 382 & $259: 239$ & 10.00 & 124.50 & 0.896 & 0.370 \\
\hline & & & $A$ & 0.429 & 382 & $239: 259$ & -10.00 & 124.50 & -0.896 & 0.370 \\
\hline & rs12900552 & 15:22795211 & G & 0.419 & 372 & $263: 239$ & 14.00 & 126.50 & 1.245 & 0.210 \\
\hline & & & A & 0.581 & 372 & $239: 263$ & -14.00 & 126.50 & -1.245 & 0.210 \\
\hline & rs7177893 & 15:22807275 & G & 0.887 & 170 & $105: 85$ & 10.00 & 47.50 & 1.451 & 0.150 \\
\hline & & & $\mathrm{T}$ & 0.113 & 170 & $85: 105$ & -10.00 & 47.50 & -1.451 & 0.150 \\
\hline & rs8025849 & 15:22825211 & A & 0.820 & 256 & $155: 139$ & 7.00 & 74.00 & 0.814 & 0.416 \\
\hline & & & G & 0.180 & 256 & $139: 155$ & -7.00 & 74.00 & -0.814 & 0.416 \\
\hline \multirow[t]{6}{*}{ NIPA2 } & rs8028189 & 15:22849067 & $C$ & 0.273 & 318 & $220: 196$ & 12.00 & 104.00 & 1.177 & 0.203 \\
\hline & & & G & 0.727 & 318 & $196: 220$ & -12.00 & 104.00 & -1.177 & 0.203 \\
\hline & rs3812922 & 15:22866361 & $C$ & 0.780 & 289 & $198: 152$ & 22.50 & 87.75 & 2.402 & 0.016 \\
\hline & & & A & 0.220 & 289 & $152: 198$ & -22.50 & 87.75 & -2.402 & 0.016 \\
\hline & rs3785 & 15:22867866 & G & 0.628 & 261 & $190: 170$ & 10.00 & 90.00 & 1.054 & 0.292 \\
\hline & & & A & 0.372 & 261 & $170: 190$ & -10.00 & 90.00 & -1.054 & 0.292 \\
\hline \multirow[t]{12}{*}{ CYFIP1 } & rs1009153 & 15:22896157 & G & 0.456 & 373 & $267: 229$ & 19.50 & 124.75 & 1.746 & 0.081 \\
\hline & & & A & 0.544 & 373 & $229: 267$ & -19.50 & 124.75 & -1.746 & 0.081 \\
\hline & rs2289818 & 15:22912200 & G & 0.588 & 353 & $254: 211$ & 20.50 & 116.75 & 1.897 & 0.058 \\
\hline & & & $C$ & 0.412 & 353 & $211: 254$ & -20.50 & 116.75 & -1.897 & 0.058 \\
\hline & rs2289823 & 15:22945116 & $C$ & 0.907 & 113 & $67: 55$ & 6.50 & 30.75 & 1.172 & 0.241 \\
\hline & & & $\mathrm{T}$ & 0.093 & 113 & $55: 67$ & -6.50 & 30.75 & -1.172 & 0.241 \\
\hline & rs12441090 & 15:22967498 & G & 0.720 & 318 & $205: 191$ & 6.50 & 99.25 & 0.625 & 0.514 \\
\hline & & & $A$ & 0.280 & 318 & $191: 205$ & -6.50 & 99.25 & -0.625 & 0.514 \\
\hline & rs8025779 & 15:22979151 & $C$ & 0.650 & 347 & $238: 220$ & 8.50 & 114.75 & 0.793 & 0.427 \\
\hline & & & G & 0.350 & 347 & $220: 238$ & -8.50 & 114.75 & -0.793 & 0.427 \\
\hline & rs8042900 & 15:22980985 & G & 0.169 & 245 & $145: 142$ & 1.50 & 71.75 & 0.177 & 0.859 \\
\hline & & & A & 0.831 & 245 & $142: 145$ & -1.50 & 71.75 & -0.177 & 0.859 \\
\hline \multirow[t]{4}{*}{ SNRPN } & rs8036523 & 15:24824408 & G & 0.227 & 300 & $179: 177$ & 0.50 & 89.25 & 0.053 & 0.958 \\
\hline & & & $\mathrm{T}$ & 0.773 & 300 & $177: 179$ & -0.50 & 89.25 & -0.053 & 0.958 \\
\hline & rs8037745 & 15:24902158 & $A$ & 0.847 & 226 & $155: 109$ & 23.00 & 66.00 & 2.831 & 0.0046 \\
\hline & & & G & 0.153 & 226 & $109: 155$ & -23.00 & 66.00 & -2.831 & 0.0046 \\
\hline \multirow[t]{6}{*}{ SNURF } & rs220030 & 15:24954621 & $\mathrm{T}$ & 0.487 & 376 & $254: 236$ & 8.50 & 122.75 & 0.767 & 0.443 \\
\hline & & & $C$ & 0.513 & 376 & $236: 254$ & -8.50 & 122.75 & -0.767 & 0.443 \\
\hline & rs2732025 & 15:24966663 & G & 0.485 & 390 & $264: 246$ & 8.50 & 127.75 & 0.752 & 0.452 \\
\hline & & & $\mathrm{T}$ & 0.514 & 390 & $246: 264$ & -8.50 & 127.75 & -0.752 & 0.452 \\
\hline & rs2732026 & 15:24971341 & A & 0.536 & 369 & $245: 235$ & 5.00 & 120.00 & 0.456 & 0.648 \\
\hline & & & $C$ & 0.464 & 369 & $235: 245$ & -5.00 & 120.00 & -0.456 & 0.648 \\
\hline \multirow[t]{2}{*}{ UBE3A } & rs8179187 & 15:25407179 & T & 0.635 & 363 & $242: 237$ & 2.00 & 120.00 & 0.183 & 0.855 \\
\hline & & & G & 0.365 & 363 & $237: 242$ & -2.00 & 120.00 & -0.183 & 0.855 \\
\hline
\end{tabular}


Table 1 continued

\begin{tabular}{|c|c|c|c|c|c|c|c|c|c|c|}
\hline Gene symbol & Marker & Chromosome & Allele & Afreq & Fam & $T: U^{a}$ & S-E (S) & $\operatorname{Var}(\mathrm{S})$ & $Z$ & $P^{\mathrm{b}}$ \\
\hline & rs2158306 & $15: 25435414$ & $\mathrm{~T}$ & 0.366 & 338 & $232: 227$ & 2.50 & 114.75 & 0.233 & 0.815 \\
\hline & & & C & 0.634 & 338 & $227: 232$ & -2.50 & 114.75 & -0.233 & 0.815 \\
\hline \multirow[t]{12}{*}{ ATP10A } & rs12324063 & 15:25743809 & G & 0.601 & 337 & $240: 207$ & 16.00 & 112.00 & 1.512 & 0.131 \\
\hline & & & A & 0.399 & 337 & $207: 240$ & -16.00 & 112.00 & -1.512 & 0.131 \\
\hline & rs4906771 & 15:25787248 & C & 0.487 & 363 & $270: 212$ & 28.00 & 121.50 & 2.540 & 0.011 \\
\hline & & & $\mathrm{T}$ & 0.513 & 363 & $212: 270$ & -28.00 & 121.50 & -2.540 & 0.011 \\
\hline & rs1345098 & 15:25812475 & $\mathrm{T}$ & 0.796 & 282 & $181: 141$ & 18.50 & 81.25 & 2.052 & 0.040 \\
\hline & & & G & 0.204 & 282 & $141: 181$ & -18.50 & 81.25 & -2.052 & 0.040 \\
\hline & rs11638520 & 15:25828376 & $\mathrm{T}$ & 0.532 & 361 & $250: 223$ & 12.50 & 118.75 & 1.147 & 0.251 \\
\hline & & & G & 0.468 & 361 & $223: 250$ & -12.50 & 118.75 & -1.147 & 0.251 \\
\hline & rs73356452 & $15: 25865066$ & C & 0.883 & 179 & $102: 100$ & 0.00 & 51.00 & 0.000 & 1.000 \\
\hline & & & $\mathrm{T}$ & 0.117 & 179 & $100: 102$ & 0.00 & 51.00 & 0.000 & 1.000 \\
\hline & rs1511488 & $15: 25866774$ & G & 0.927 & 125 & $73: 59$ & 7.00 & 33.00 & 1.219 & 0.223 \\
\hline & & & C & 0.073 & 125 & $59: 73$ & -7.00 & 33.00 & -1.219 & 0.223 \\
\hline \multirow[t]{16}{*}{ GABRB3 } & rs12438141 & 15:26625455 & $C$ & 0.922 & 131 & $86: 60$ & 13.00 & 36.50 & 2.152 & 0.031 \\
\hline & & & $\mathrm{T}$ & 0.078 & 131 & $60: 86$ & -13.00 & 36.50 & -2.152 & 0.031 \\
\hline & rs9806546 & $15: 26648239$ & A & 0.833 & 242 & $150: 133$ & 8.00 & 71.00 & 0.949 & 0.343 \\
\hline & & & G & 0.167 & 242 & $133: 150$ & -8.00 & 71.00 & -0.949 & 0.343 \\
\hline & rs1863467 & 15:26688587 & C & 0.574 & 346 & $253: 202$ & 24.50 & 114.25 & 2.292 & 0.022 \\
\hline & & & $\mathrm{T}$ & 0.426 & 346 & $202: 253$ & -24.50 & 114.25 & -2.292 & 0.022 \\
\hline & rs7180158 & 15:26733091 & A & 0.359 & 341 & $224: 209$ & 7.50 & 108.25 & 0.721 & 0.471 \\
\hline & & & G & 0.641 & 341 & $209: 224$ & -7.50 & 108.25 & -0.721 & 0.471 \\
\hline & rs12593579 & 15:26742985 & A & 0.689 & 350 & $224: 215$ & 5.00 & 110.00 & 0.477 & 0.634 \\
\hline & & & $C$ & 0.311 & 350 & $215: 224$ & -5.00 & 110.00 & -0.477 & 0.634 \\
\hline & rs7165604 & 15:26749309 & $\mathrm{T}$ & 0.684 & 286 & $192: 186$ & 3.00 & 94.50 & 0.309 & 0.758 \\
\hline & & & C & 0.316 & 286 & $186: 192$ & -3.00 & 94.50 & -0.309 & 0.758 \\
\hline & rs8041610 & 15:26763117 & C & 0.414 & 378 & $253: 246$ & 3.00 & 125.50 & 0.268 & 0.789 \\
\hline & & & A & 0.586 & 378 & $246: 253$ & -3.00 & 125.50 & -0.268 & 0.789 \\
\hline & rs4906902 & 15:26774621 & A & 0.667 & 342 & $263: 197$ & 33.00 & 115.00 & 3.077 & 0.002 \\
\hline & & & G & 0.333 & 342 & $197: 263$ & -33.00 & 115.00 & -3.077 & 0.002 \\
\hline \multirow[t]{2}{*}{ GABRA5 } & rs35586628 & 15:26886993 & $\mathrm{T}$ & 0.595 & 366 & $254: 226$ & 14.00 & 120.00 & 1.278 & 0.201 \\
\hline & & & C & 0.405 & 366 & $226: 254$ & -14.00 & 120.00 & -1.278 & 0.201 \\
\hline \multirow[t]{7}{*}{ GABRG3 } & rs7403021 & 15:26970515 & C & 0.815 & 252 & $158: 147$ & 5.50 & 76.25 & 0.63 & 0.529 \\
\hline & & & $\mathrm{T}$ & 0.185 & 252 & $147: 158$ & -5.50 & 76.25 & -0.63 & 0.529 \\
\hline & rs7180500 & 15:27008032 & C & 0.904 & 159 & $112: 66$ & 23.00 & 44.50 & 3.488 & 0.00057 \\
\hline & & & A & 0.096 & 159 & $66: 112$ & -23.00 & 44.50 & -3.488 & 0.00057 \\
\hline & rs12910555 & 15:27122424 & A & 0.872 & 195 & $120: 102$ & 8.50 & 55.75 & 1.138 & 0.255 \\
\hline & & & G & 0.128 & 195 & $102: 120$ & -8.50 & 55.75 & -1.138 & 0.255 \\
\hline & rs897173 & $15: 27224754$ & A & 0.581 & 371 & $263: 229$ & 17.00 & 123.50 & 1.530 & 0.126 \\
\hline
\end{tabular}


Table 1 continued

\begin{tabular}{|c|c|c|c|c|c|c|c|c|c|c|}
\hline Gene symbol & Marker & Chromosome & Allele & Afreq & Fam & $T: U^{a}$ & S-E (S) & $\operatorname{Var}(\mathrm{S})$ & $Z$ & $P^{\mathrm{b}}$ \\
\hline & & & G & 0.419 & 371 & $229: 263$ & -17.00 & 123.50 & -1.530 & 0.126 \\
\hline & rs4778159 & 15:27396841 & A & 0.684 & 322 & $217: 177$ & 19.50 & 98.75 & 1.962 & 0.050 \\
\hline & & & T & 0.316 & 322 & $177: 217$ & -19.50 & 98.75 & -1.962 & 0.050 \\
\hline & rs140679 & 15:27527530 & T & 0.687 & 322 & $214: 199$ & 6.50 & 103.75 & 0.638 & 0.523 \\
\hline & & & C & 0.313 & 322 & $199: 214$ & -6.50 & 103.75 & -0.638 & 0.523 \\
\hline
\end{tabular}

Afreq allele frequency, Fam number of informative families, $S$ test statistics for the observed number of transmitted alleles, $E(S)$ expected value of $S$ under the null hypothesis (i.e., no linkage and no association)

${ }^{a}$ The ratio of transmisson to untransmisson $(T: U)$ for each selected SNP was calculated by the Haploveiw version 4.2

${ }^{\mathrm{b}} P$-value with bold character means the statistical significance persists after the Bonferroni correction

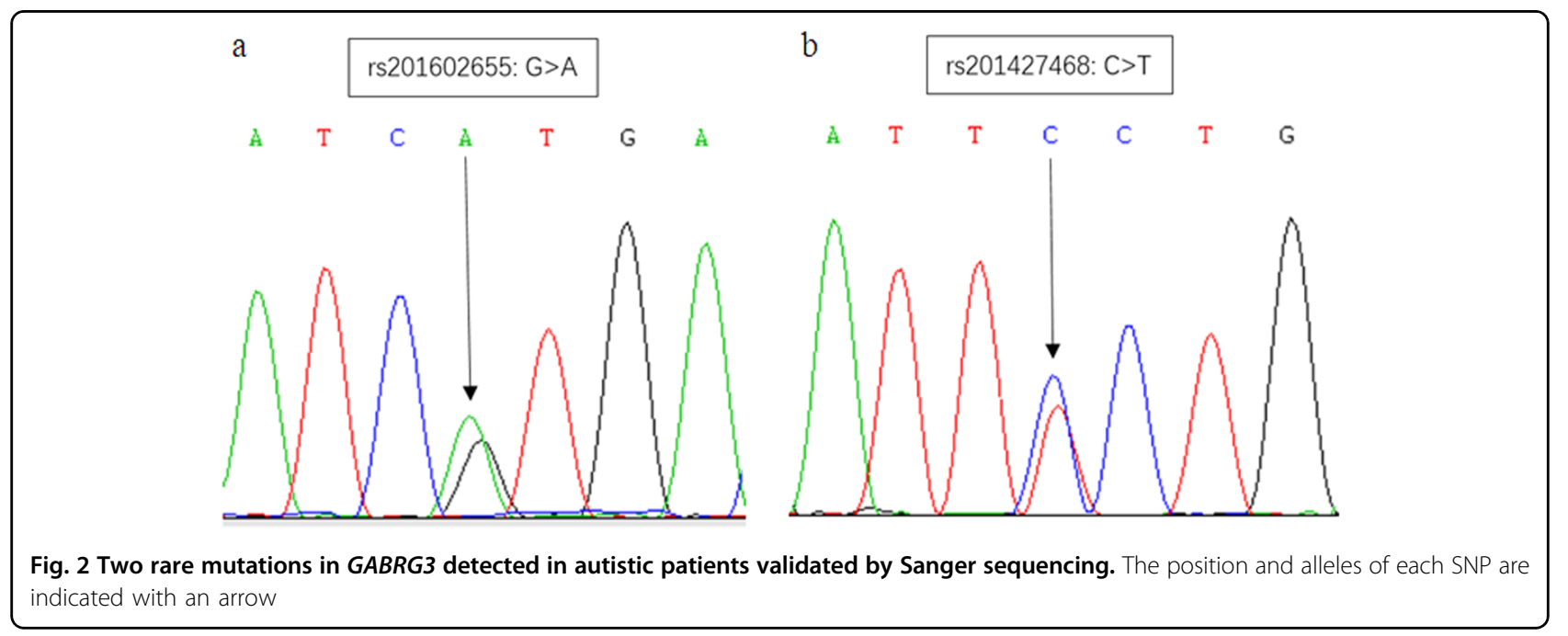

stages. After birth, the expression of GABRG3 was stabilized at a relatively high level in different regions of the brain throughout life (Fig. S5a). The dynamic expression of GABRG3 in 11 areas of the neocortex was similar to the expression in other brain regions (Fig. S5b).

\section{Function analysis of the associated SNPs and sequenced variants}

The function prediction revealed that the rs7180500, rs201602655 (p.Val233Met) and rs201427468 (p.Pro365Ser) in GABRG3 might alter the regulatory motifs, as well as rs4906902 in GABRB3 and rs4906771 in ATP10A (Table S8). Additionally, three programs (Polyphen2, SIFT and Mutation Taster) predicted that rs201602655 (p. Val233Met) in GABRG3 might be deleterious and affect protein function (Table 2). The rs201427468 (p.Pro365Ser) in GABRB3 was predicted to be damaging only by the Mutation Taster software.

\section{Discussion}

Our study indicated that the C allele of rs7180500 in GABRG3 was significantly associated with autism in 512 autism trios. The eQTL data from two web sources confirmed that this risk allele might be correlated with the expression of GABRG3 in the cerebellum. Moreover, we sequenced the genes on chromosome 15q11-q13 region and identified two rare variants rs201602655 (p.Val233Met) and rs201427468 (p.Pro365Ser) in GABRG3 and six rare variants in $G A B R B 3$. Among these mutations, the frequency of rs201602655 (p.Val233Met) in GABRG3 in autistic children was significantly higher than that in healthy controls $\left(9 / 512\right.$ vs. $2 / 575$, Pearson $\chi^{2}$-test $\chi^{2}=$ $5.375, p=0.020$ ). The function prediction indicated that rs201602655 (p.Val233Met) might be deleterious. Additionally, rs4906902 in GABRB3 and rs4906771 in ATP10A exhibited significant association with autism under the recessive model.

In the Psychiatry Genomics Consortium (PGC) ASD subset (available at: http://www.med.unc.edu/pgc/resultsand-downloads), rs7180500 in GABRG3 was nominally associated with autism $(\mathrm{OR}=0.938 ; \mathrm{SE}=0.028 ; p=$ 0.021) (Table S5), whereas rs4906902 in GABRB3 and rs4906771 in $A T P 10 A$ did not show a significant association. Other significant risk alleles reported in 
Caucasians, such as rs7180158, rs7165604, rs12593579 and rs9806546 in GABRB3, did not exhibit a positive association with autism in our samples. Moreover, our study demonstrated that rs4906902 in GABRB3 and rs4906771 in $A T P 10 A$ were significantly associated with autism under the recessive model, although these positive results were not replicated in ASD subset of PGC. In addition, a previous study reported no significant association between GABA receptor genes on chromosome 15q11-q13 and autism in 166 Japanese autistic patients and 412 controls after Bonferroni correction. However, nominal significant associatin of rs3212337 in GABRB3 and rs4887536 in GABRG3 and autism were observed ${ }^{46}$. It is suggested that further search for susceptibility variants should be performed.

Our results were inconsistent with PGC autism data and previous study in Japanese population, which might result from a few reasons: First, ethnic heterogeneity might be considered. Due to different genetic backgrounds, the MAF of rs7180500 in our samples and the East Asian population was approximately 0.10, while 0.49 in Europeans. Second, ASD is a genetic heterogenous spectrum. Subjects of other studies were mostly ASD individuals. To decrease heterogeneity, our study recruited families with classical autistic individuals. Third, the genetic signal might be tagging other genetic variants, which directly contribute to the autism risk.

We detected a rare variant rs201602655 (p.Val233Met) in 9 of 512 autistic patients. The frequency of rs201602655 (p.Val233Met) in GABRG3 in autistic children was significantly higher than that in healthy children. GABRG3 had found to be intolerant to the heterozygous missense variant based on the $Z$-score of 1.92 from the Exome Aggregation Consortium (ExAC) database. This indicates that the detected missense variants might be deleterious. Among the 9 patients carrying rs201602655 (p.Val233Met), one patients carried the de novo variant, and the other 8 patients were inherited this variant from their parents (all heterozygotes). Previous studies showed that in the low-risk families, the de novo mutations might contribute to the development of autism. On the other hand, in high-risk families, the inherited variants might increase the risk and susceptibility to autism ${ }^{47,48}$. Heritability estimates strongly support a significant role for autosomal inherited factors ${ }^{2,49}$. Indeed, combined variants including rare de novo and inherited variants were reported to be affected in the case to reach the threshold for a fully penetrant phenotype, suggesting a 'multiple hit' model of $\mathrm{ASD}^{50-52}$. Our results might indicate that the rare variants detected in GABRG3 might contribute to the increased risk of autism.

Autism is a complex disorder with high heritability and heterogeneity. Both common and rare variations contribute to liability. The common variants increase the risk 


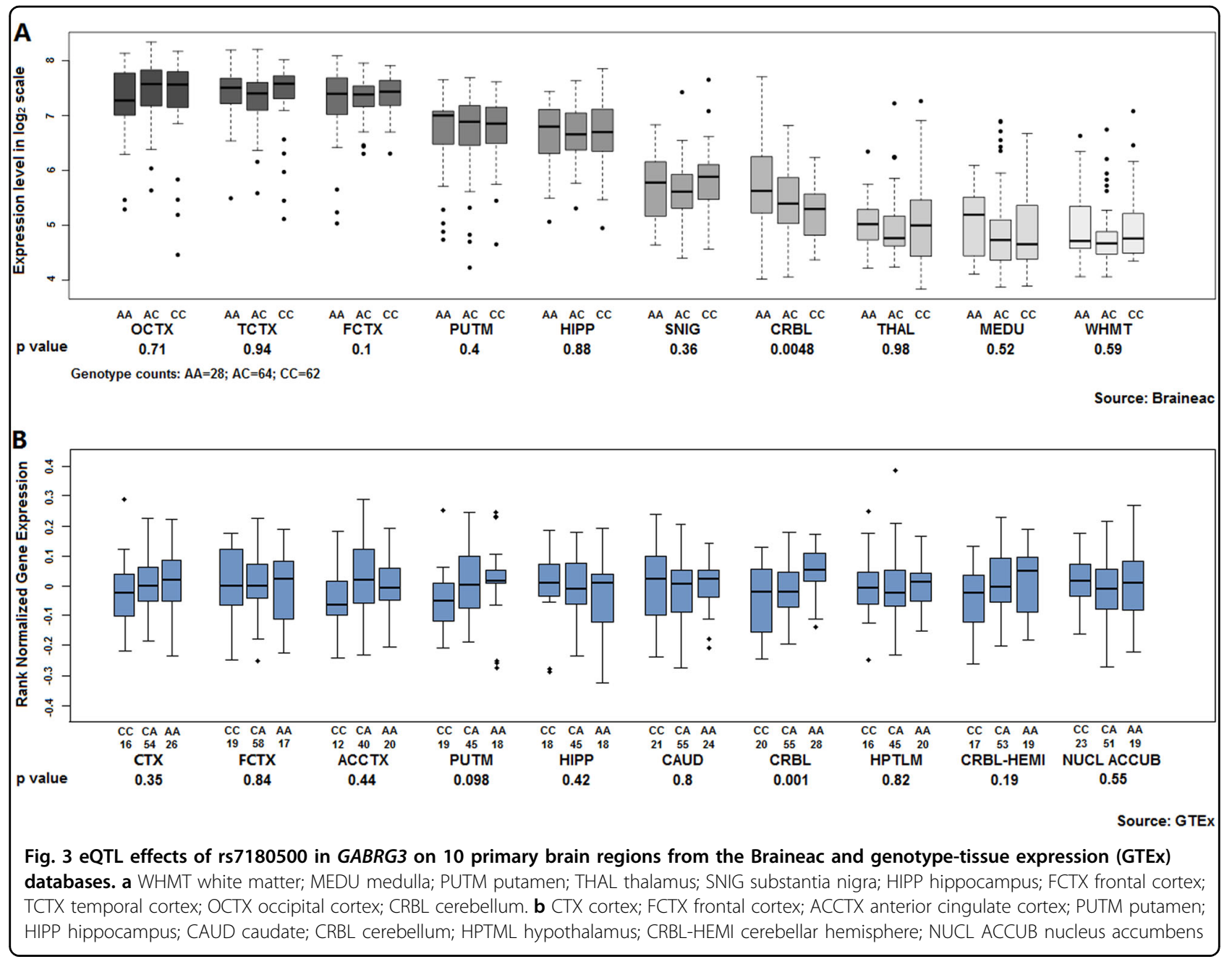

for autism with a small effect, and the interaction with other susceptibility genes and environmental factors might underlie the pathogenesis of autism. Although the frequencies of rare variants were low, they might exhibit loss of function (LoF) effect that could be the causative factor for autism. Recent studies have identified distinct and individually rare genetic causes, suggesting that the genetic architecture of autism might contribute significantly to heterogeneous rare variants ${ }^{28,48,53,54}$. In this study, we detected that both common variant (rs7180500) and rare variant rs201602655 (p.Val233Met) in GABRG3 were associated with autism in Chinese Han population. Our study provided new evidence for the contribution of common and rare variations to the etiology of autism. The potential risk of common variants and rare mutations in GABRG3 remained to be explored in different ethnic populations using large sample studies.

Furthermore, the functional evidence indicated that abnormal expression of GABRG3 and other $\mathrm{GABA}_{\mathrm{A}}$ receptors subunits genes could serve as susceptibility factors for autism. Fatemi et al. further found an abnormal expression of GABRG3 in the cerebellum and other Brodmann areas of autistic individuals ${ }^{8}$. The current study showed that the risk $C$ allele of rs7180500 might lead to a lower expression of GABRG3 in human cerebellum by eQTL data analysis, which might partially trigger abnormal social phenotypes. Several studies suggested that the dysregulation of GABAergic transmission and an imbalance between excitation and inhibition (E/I) in the selective neuronal circuits in the brain of autistic individuals might be involved in social and emotional processes ${ }^{55,56} . \mathrm{GABA}_{\mathrm{A}}$ receptors, including GABRG3, played a critical role in modulating the intracellular $\mathrm{Ca}^{2+}$ concentration during different developmental stages. Along with the maturation of the central nervous system, the increase in intracellular $\mathrm{Ca}^{2+}$ concentration was gradually reduced via the regulation of $\mathrm{GABA}_{\mathrm{A}}$ receptors ${ }^{5}$. Moreover, GABRG3 contained benzodiazapine binding sites. Some pharmacological studies indicated that reduced benzodiazepine binding sites in $\mathrm{GABA}_{\mathrm{A}}$ receptors were observed in several brain regions of autistic individuals ${ }^{57-59}$. Therefore, $\mathrm{GABA}_{\mathrm{A}}$ receptor genes 
cluster including GABRG3 might play a role in the etiology of autism.

Our study has a few limitations. The other genes located on 15q11-13 need to be studied. The $15 \mathrm{q} 13.3$ microdeletion syndrome is associated with numerous conditions, including ASD, epilepsy, schizophrenia, and intellectual disability ${ }^{6-64}$. Deletion of genes located in this region, such as CHRNA7 and OTUD7A was responsible for the $15 \mathrm{q} 13.3$ microdeletion syndrome ${ }^{65,66}$. Recent studies indicated that disruption of OTUD7A could cause neurodevelopmental deficits including abnormal cortical neuron morphology, which recapitulated some of the characteristics of the $15 \mathrm{q} 13.3$ microdeletion syndrome $^{67,68}$. Therefore, genetic studies on the relationship of variants in candidate genes such as CHRNA7 and OTUD7A on 15q11-q13 with autism should be performed.

Our study suggested that common variants and rare variants in GABRG3 were significantly associated with autism. GABRG3 might contribute to the pathogenesis of autism. Moreover, GABRB3 and ATP10A located on chromosome 15q11-q13 might increase risk for autism in Chinese Han population.

\section{Acknowledgements}

We thank all participants and their families for their participation in this study. This work was supported by grants from the National Key R\&D Program of China (2017YFC1309901), National Natural Science Foundation of China (grant numbers 81471383, 81471360, 81501183, and 81730037).

\section{Author details \\ ${ }^{1}$ Peking University Sixth Hospital, Beijing 100191, China. ${ }^{2}$ Peking University Institute of Mental Health, Beijing 100191, China. ${ }^{3}$ Key Laboratory of Mental Health, Ministry of Health (Peking University), Beijing 100191, China. ${ }^{4}$ National Clinical Research Center for Mental Disorders, (Peking University Sixth Hospital), Beijing 100191, China. ${ }^{5}$ The Affiliated Brain Hospital of Guangzhou Medical University (Guangzhou Huiai Hospital), Guangzhou 510370, China. ${ }^{6}$ Peking- Tsinghua Center for Life Sciences, Peking University, Beijing 100871, China. ${ }^{7}$ PKU-IDG/McGovern Institute for Brain Research, Peking University, Beijing 100871, China}

\section{Author contributions}

L.F.W., Z.L.W., and D.Z. designed the study. L.Y.W., J.Li., Z.L.W., M.S., T.L.L., Z.Q.W. T.Z., and Y.Y.R. performed the experiments. J.Li., Z.L.W., L.Y.W., and W.H.Y. analyzed the data. M.X.J., L.F.W., and M.S. collected the samples. L.Y.W., J.Li., Z.L. W., L.F.W., Z.Q.W., T.Z., D.Z., and J. Liu wrote the paper and revised it. All authors reviewed the manuscript and approved the submission.

\section{Conflict of interest}

The authors declare that they have no conflict of interest.

\section{Publisher's note}

Springer Nature remains neutral with regard to jurisdictional claims in published maps and institutional affiliations.

Supplementary Information accompanies this paper at (https://doi.org/ 10.1038/s41398-018-0197-4).

Received: 10 March 2018 Revised: 25 May 2018 Accepted: 8 June 2018 Published online: 14 August 2018

\section{References}

1. Sandin, S. et al. The familial risk of autism. JAMA 311, 1770-1777 (2014).

2. Bailey, A. et al. Autism as a strongly genetic disorder - evidence from a British Twin Study. Psychol. Med. 25, 63-77 (1995).

3. De Rubeis, S. \& Buxbaum, J. D. Recent advances in the genetics of Autism spectrum disorder. Curr. Neurol. Neurosci. Rep. 15, 36 (2015).

4. Devlin, B. \& Scherer, S. W. Genetic architecture in autism spectrum disorder. Curr. Opin. Genet. Dev. 22, 229-237 (2012).

5. Li, K. \& Xu, E. The role and the mechanism of gamma-aminobutyric acid during central nervous system development. Neurosci. Bull. 24, 195-200 (2008).

6. Valluy, J. et al. A coding-independent function of an alternative Ube3a transcript during neuronal development. Nat. Neurosci. 18, 666-673 (2015).

7. Hsiao, K. et al. Cyfip1 regulates presynaptic activity during development. J. Neurosci. 36, 1564-1576 (2016).

8. Fatemi, S. H. \& Folsom, T. D. GABA receptor subunit distribution and FMRPmGluR5 signaling abnormalities in the cerebellum of subjects with schizophrenia, mood disorders, and autism. Schizophr. Res. 167, 42-56 (2015).

9. Mendez, M. A. et al. The brain GABA-benzodiazepine receptor alpha-5 subtype in autism spectrum disorder: a pilot [(11)C]Ro15-4513 positron emission tomography study. Neuropharmacology 68, 195-201 (2013).

10. DeLorey, T. M. et al. Gabrb3 gene deficient mice exhibit impaired social and exploratory behaviors, deficits in non-selective attention and hypoplasia of cerebellar vermal lobules: a potential model of autism spectrum disorder. Behav. Brain. Res. 187, 207-220 (2008).

11. DeLorey, T. M. et al. Somatosensory and sensorimotor consequences associated with the heterozygous disruption of the autism candidate gene, Gabrb3. Behav. Brain Res. 216, 36-45 (2011).

12. Zurek, A. A. et al. alpha5GABAA receptor deficiency causes autism-like behaviors. Ann. Clin. Transl. Neurol. 3, 392-398 (2016).

13. Mesbah-Oskui, L., Penna, A., Orser, B. A. \& Horner, R. L. Reduced expression of alpha5GABAA receptors elicits autism-like alterations in EEG patterns and sleep-wake behavior. Neurotoxicol. Teratol. 61, 115-122 (2017).

14. Smith, S. E. et al. Increased gene dosage of Ube3a results in autism traits and decreased glutamate synaptic transmission in mice. Sci. Transl. Med. 3, 103ra197 (2011).

15. $\mathrm{Li}, \mathrm{H}$. et al. The autism-related gene SNRPN regulates cortical and spine development via controlling nuclear receptor Nr4a1. Sci. Rep. 6, 29878 (2016).

16. Oguro-Ando, A. et al. Increased CYFIP1 dosage alters cellular and dendritic morphology and dysregulates mTOR. Mol. Psychiatry 20, 1069-1078 (2015).

17. Pathania, M. et al. The autism and schizophrenia associated gene CYFIP1 is critical for the maintenance of dendritic complexity and the stabilization of mature spines. Transl. Psychiatry 4, e374 (2014).

18. Martinez-Cerdeno, V. Dendrite and spine modifications in autism and related neurodevelopmental disorders in patients and animal models. Dev. Neurobiol. 77, 393-404 (2017)

19. Menold, M. M. et al. Association analysis of chromosome 15 gabaa receptor subunit genes in autistic disorder. J. Neurogenet. 15, 245-259 (2001).

20. McCauley, J. L. et al. A linkage disequilibrium map of the 1-Mb 15q12 GABA(A) receptor subunit cluster and association to autism. Am. J. Med. Genet. B. Neuropsychiatr. Genet. 131B, 51-59 (2004).

21. Kim, S. J. et al. Transmission disequilibrium testing of the chromosome 15q11q13 region in autism. Am. J. Med. Genet. B. Neuropsychiatr. Genet. 147B, 1116-1125 (2008)

22. Kim, S. A. et al. Association of GABRB3 polymorphisms with autism spectrum disorders in Korean trios. Neuropsychobiology 54, 160-165 (2006).

23. Warrier, V., Baron-Cohen, S. \& Chakrabarti, B. Genetic variation in GABRB3 is associated with Asperger syndrome and multiple endophenotypes relevant to autism. Mol. Autism 4, 48 (2013).

24. Waltes, R. et al. Common variants in genes of the postsynaptic FMRP signalling pathway are risk factors for autism spectrum disorders. Hum. Genet. 133, 781-792 (2014).

25. Wang, J. et al. Common regulatory variants of CYFIP1 contribute to susceptibility for Autism Spectrum Disorder (ASD) and Classical Autism. Ann. Hum. Genet. 79, 329-340 (2015).

26. Nurmi, E. L. et al. Dense linkage disequilibrium mapping in the 15q11-q13 maternal expression domain yields evidence for association in autism. Mol. Psychiatry 8, 624-634 (2003).

27. Bandyopadhyay, B., Chanda, V. \& Wang, Y. Finding the sources of missing heritability within rare variants through simulation. Bioinform. Biol. Insights $\mathbf{1 1}$ 1177932217735096 (2017). 
28. Sanders, S. J. et al. De novo mutations revealed by whole-exome sequencing are strongly associated with autism. Nature 485, 237-241 (2012).

29. Neale, B. M. et al. Patterns and rates of exonic de novo mutations in autism spectrum disorders. Nature 485, 242-245 (2012)

30. Iossifov, I. et al. De novo gene disruptions in children on the autistic spectrum. Neuron 74, 285-299 (2012)

31. Picinelli, C. et al. Recurrent 15q11.2 BP1-BP2 microdeletions and microduplications in the etiology of neurodevelopmental disorders. Am. J. Med. Genet. B. Neuropsychiatr. Genet. 171, 1088-1098 (2016).

32. van der Zwaag, B. et al. A co-segregating microduplication of chromosome $15 q 11.2$ pinpoints two risk genes for autism spectrum disorder. Am. J. Med. Genet. B. Neuropsychiatr. Genet. 153B, 960-966 (2010).

33. Chen, C. H. et al. Genetic analysis of GABRB3 as a candidate gene of autism spectrum disorders. Mol. Autism 5, 36 (2014).

34. Delahanty, R. J. et al. Maternal transmission of a rare GABRB3 signal peptide variant is associated with autism. Mol. Psychiatry 16, 86-96 (2011).

35. Krug, D. A., Arick, J. \& Almond, P. Behavior checklist for identifying severely handicapped individuals with high levels of autistic behavior. J. Child Psychol. Psychiatry 21, 221-229 (1980).

36. Schopler, E., Reichler, R. J., DeVellis, R. F. \& Daly, K. Toward objective classification of childhood autism: Childhood Autism Rating Scale (CARS). J. Autism Dev. Disord. 10, 91-103 (1980).

37. Storm, N., Darnhofer-Patel, B., van den Boom, D. \& Rodi, C. P. MALDI-TOF mass spectrometry-based SNP genotyping. Methods Mol. Biol. 212, 241-262 (2003).

38. Jurinke, C., van den Boom, D., Cantor, C. R. \& Koster, H. The use of MassARRAY technology for high throughput genotyping. Adv. Biochem. Eng. Biotechnol. 77, 57-74 (2002).

39. Li, H. \& Durbin, R. Fast and accurate long-read alignment with BurrowsWheeler transform. Bioinformatics 26, 589-595 (2010).

40. McKenna, A. et al. The Genome Analysis Toolkit: a MapReduce framework for analyzing next-generation DNA sequencing data. Genome Res. 20, 1297-1303 (2010).

41. Kazeem, G. R. \& Farrall, M. Integrating case-control and TDT studies. Ann. Hum. Genet. 69, 329-335 (2005).

42. Ramasamy, A. et al. Genetic variability in the regulation of gene expression in ten regions of the human brain. Nat. Neurosci. 17, 1418-1428 (2014).

43. Consortium, G. T. The Genotype-Tissue Expression (GTEx) project. Nat. Genet. 45, 580-585 (2013).

44. Ward, L. D. \& Kellis, M. HaploReg: a resource for exploring chromatin states, conservation, and regulatory motif alterations within sets of genetically linked variants. Nucleic Acids Res. 40, D930-D934 (2012).

45. Guo, L., Du, Y., Qu, S. \& Wang, J. rVarBase: an updated database for regulatory features of human variants. Nucleic Acids Res. 44, D888-D893 (2016).

46. Tochiqi, $M$. et al. No evidence for significant association between GABA receptor genes in chromosome $15 q 11-q 13$ and autism in a Japanese population. J. Hum. Genet. 52, 985-989 (2007).

47. Zhao, X. et al. A unified genetic theory for sporadic and inherited autism. Proc. Natl Acad. Sci. USA 104, 12831-12836 (2007).

48. Ronemus, M., lossifov, I., Levy, D. \& Wigler, M. The role of de novo mutations in the genetics of autism spectrum disorders. Nat. Rev. Genet. 15, 133-141 (2014).

49. Hallmayer, J. et al. Genetic heritability and shared environmental factors among twin pairs with autism. Arch. Gen. Psychiatry 68, 1095-1102 (2011).
50. Berkel, S. et al. Inherited and de novo SHANK2 variants associated with autism spectrum disorder impair neuronal morphogenesis and physiology. Hum. Mol. Genet. 21, 344-357 (2012).

51. Girirajan, S. et al. A recurrent 16p12.1 microdeletion supports a two-hit model for severe developmental delay. Nat. Genet. 42, 203-209 (2010).

52. Leblond, C. S. et al. Genetic and functional analyses of SHANK2 mutations suggest a multiple hit model of autism spectrum disorders. PLoS Genet. 8, e1002521 (2012).

53. O'Roak, B. J. et al. Exome sequencing in sporadic autism spectrum disorders identifies severe de novo mutations. Nat. Genet. 43, 585-589 (2011).

54. Pagnamenta, A. T. et al. Exome sequencing can detect pathogenic mosaic mutations present at low allele frequencies. J. Hum. Genet. 57, 70-72 (2012).

55. Cellot, G. \& Cherubini, E. GABAergic signaling as therapeutic target for autism spectrum disorders. Front. Pediatr. 2, 70 (2014).

56. Bonnet-Brilhault, F. et al. GABA/Glutamate synaptic pathways targeted by integrative genomic and electrophysiological explorations distinguish autism from intellectual disability. Mol. Psychiatry 21, 411-418 (2016).

57. Hadingham, K. L. et al. Expression and pharmacology of human GABAA receptors containing gamma 3 subunits. Eur. J. Pharmacol. 291, 301-309 (1995).

58. Oblak, A., Gibbs, T. T. \& Blatt, G. J. Decreased GABAA receptors and benzodiazepine binding sites in the anterior cingulate cortex in autism. Autism Res. 2 , 205-219 (2009).

59. Oblak, A. L., Gibbs, T. T. \& Blatt, G. J. Reduced GABAA receptors and benzodiazepine binding sites in the posterior cingulate cortex and fusiform gyrus in autism. Brain Res. 1380, 218-228 (2011).

60. Sharp, A. J. et al. A recurrent $15 q 13.3$ microdeletion syndrome associated with mental retardation and seizures. Nat. Genet. 40, 322-328 (2008).

61. Ben-Shachar, S. et al. Microdeletion 15q13.3: a locus with incomplete penetrance for autism, mental retardation, and psychiatric disorders. J. Med. Genet. 46, 382-388 (2009).

62. Helbig, l. et al. 15q13.3 microdeletions increase risk of idiopathic generalized epilepsy. Nat. Genet. 41, 160-162 (2009).

63. Shinawi, M. et al. A small recurrent deletion within $15 q 13.3$ is associated with a range of neurodevelopmental phenotypes. Nat. Genet. 41, 1269-1271 (2009).

64. Lowther, $\mathrm{C}$. et al. Delineating the $15 \mathrm{q} 13.3$ microdeletion phenotype: a case series and comprehensive review of the literature. Genet. Med. 17, 149-157 (2014).

65. Hoppman-Chaney, N. et al. Homozygous deletion of chromosome 15q13.3 including CHRNA7 causes severe mental retardation, seizures, muscular hypotonia, and the loss of KLF13 and TRPM1 potentially cause macrocytosis and congenital retinal dysfunction in siblings. Eur. J. Med. Genet. 54, e441-e445 (2011).

66. Hoppman-Chaney, N. et al. Identification of single gene deletions at 15q13.3: further evidence that CHRNA7 causes the 15q13.3 microdeletion syndrome phenotype. Clin. Genet. 83, 345-351 (2013).

67. Uddin, M. et al. OTUD7A regulates neurodevelopmental phenotypes in the $15 q 13.3$ microdeletion syndrome. Am. J. Hum. Genet. 102, 278-295 (2018).

68. Yin, J. et al. Otud7a knockout mice recapitulate many neurological features of 15q13.3 microdeletion syndrome. Am. J. Hum. Genet. 102, 296-308 (2018). 